\title{
Effect of Pistacia Atlantica Resin Oil on Anti- Oxidant, Hydroxyprolin and VEGF Changes in Experimentally-Induced Skin Burn in Rat
}

\author{
Beydolah Shahouzehi ${ }^{1,2^{*}}$, Gholamreza Sepehri ${ }^{3}$, Sakine Sadeghiyan ${ }^{4}$, \\ Yaser Masoomi-Ardakani ${ }^{2}$
}

1. Student Research Committee, School of Medicine, Kerman University of Medical Sciences, Kerman, Iran;

2. Physiology Research Center, Institute of Neuropharmacology, Kerman University of Medical Sciences, Kerman, Iran;

3. Department of Physiology and Pharmacology, Kerman University of Medical Sciences, Kerman, Iran;

4. Neuroscience Research Center, Kerman University of Medical Sciences, Kerman, Iran

*Corresponding Author:

Beydolah Shahouzehi, PhD;

Department of Clinical Biochemistry, Boulevard Jihad, Ebn-e-Sina Avenue, Postal code: 7619813159,

Kerman, Iran.

Tel: +98-341-2236839

Fax: +98-341-2264097.

Email: bshahouzehi@yahoo.com, bshahouzehi@gmail.com

Received: November 2, 2017

Revised: July 30, 2018

Accepted: August 19, 2018

\section{ABSTRACT}

\section{BACKGROUND}

Severe burn damage and its consequences are life threatening which can complicate patients' health. Medicinal and traditional plants are considered as safe, natural and inexpensive source of treatment for wide variety of diseases. This study assessed beneficial effect of Pistacia atlantica oil on rats burn wound healing and its potential effects on malondialdehyde (MDA), vasculoendothelial growth factor (VEGF), hydroxyprolin and antioxidant status in wound area.

\section{METHODS}

Thirty male rats weighing $200 \pm 10 \mathrm{~g}$ were randomly divided into three groups $(n=10)$ as follows. Group 1 underwent just burn injury, Group 2 underwent burn injury and received $150 \mathrm{mg} / \mathrm{kg} /$ day $P$. atlantica oil topically, and Group 3 underwent burn injury and received $150 \mathrm{mg} / \mathrm{kg} /$ day sulfadiazine cream topically. At the end of the study (day 14), wounded areas were measured and then skin in the burn damage were dissected and anti-oxidative parameter, MDA, VEGF and hydroxyprolin were evaluated.

\section{RESULTS}

P. Atlantica oil significantly increased antioxidant defense, VEGF, hydroxyprolin and reduced MDA levels. It could remarkably reduce wound size compared to burn control group. P. Atlantica oil showed more beneficial effects than sulfadiazine.

\section{CONCLUSION}

$P$. atlantica resin oil could be considered as a new therapeutic agent for treatment of injuries.

\section{KEYWORDS}

Pistacia atlantica; VEGF; Anti-oxidant; Burn injury; MDA

Please cite this paper as:

Shahouzehi B, Sepehri GR, Sadeghiyan S, Masoomi-Ardakani Y. Effect of Pistacia Atlantica Resin Oil on Anti-Oxidant, Hydroxyprolin and VEGF Changes in Experimentally-Induced Skin Burn in Rat. World J Plast Surg 2018;7(3):357-363. doi: 10.29252/wips.7.3.357.

INTRODUCTION

Burn damage and its consequences lead to major problems which can complicate patients' health. Seriously burned patients' needs 
strict regular care including wound healing care and treatment, nutritional supports and control of probable infection. ${ }^{1}$ Pathophysiological changes in burned area caused by increased tissue temperature leads to inflammatory response and also thermal exposure can cause necrosis in burn area especially in middle parts. ${ }^{2}$ Increased Reactive Oxygen Species (ROSs) lead to severe damage to the cells in burn area. ${ }^{3}$

Wound healing is a dynamic process which consist three phases including inflammation, proliferation and maturation and cytokines and reduced local ischemia and ROSs have a pivotal role in this process. ROSs are one of the components which are participates in tissue damage. Also following thermal injury ROSs have been considered to participate in a number of pathophysiological steps. It has also been reported that ROSs are involved in burn shock and lung damage following thermal injury.,2

Sepsis is the leading cause of mortality in burn units and infection is one of the major problems correlated burn injuries. Nevertheless, antibacterial remedies which used in order to eliminate infection from burn area, the infection problem still is present and need more attention. ${ }^{4}$ Vasculoendothelial Growth Factor (VEGF) is a multi-action growth factor that facilitates wound healing and helps tissue repair. VEGF increases inflammatory cells in damage area and also promotes migration and proliferation of endothelial cells. ${ }^{4,5}$

Collagen is an extracellular matrix protein which is related to wound healing and strength. Hydroxyproline is an amino acid that especially presents in collagen structure and its levels in wound parts may be as a marker of wound healing rate. ${ }^{6}$ Medicinal and traditional plants always considered as a good, safe and inexpensive source of remedy for many diseases. Therefore, a wide range of plants are used as pharmacological agent against diseases and improve body health..$^{7-10}$

It has been reported that Emu oil postpones the wound healing process at inflammatory process but on the other hand Emu oil showed beneficial wound healing effect on keratinization of epidermis. ${ }^{8}$ Other study demonstrated that Capparis spinosa leaves hydro-alcoholic extract attenuates inflammation and also promoted wound healing process. ${ }^{7}$ One of these traditional plants that used commonly is Pistacia atlantica which has been reported that have many potential beneficial effects. ${ }^{10-15} P$. atlantica is a plant which is widely distributed in Algeria, Iran, Iraq, Mediterranean and turkey. ${ }^{9}$

Its resin used as chewing gum and mouth freshener. $P$. atlantica resin have used as a traditional treatment for peptic ulcer disease. ${ }^{11}$ There are some studies about wound healing and anti-inflammatory properties of $P$. atlantica resin in animal models and also it used traditionally as a remedy for wound healing in some parts of Iran. ${ }^{10}$ Peksel et al. (2013) showed that aqueous extract of Pistacia leaves has radical scavenging properties. ${ }^{16}$ Also other studies were approved antioxidant activity of $P$. atlantica. ${ }^{12,14}$ It have been proved that $P$. atlantica have used for treatment of digestive diseases and also traditionally used for treatment of disorders such as colitis, gastrointestinal problems, kidney, heart and liver complications. ${ }^{910}$ Other studies reported antifungal, antiparasite and antibacterial activity of $P$. atlantica. ${ }^{13-15} \mathrm{In}$ present study we evaluated ameliorative effect of $P$. atlantica resin oil on wound healing in rat which burden experimentally burn wound on skin and also we assessed $P$. atlantica resin oil effects on VEGF, hydroxyproline, and antioxidant status in wound area.

\section{MATERIALS AND METHODS}

Thirty male Sprague-Dawley rats weighing $200 \pm 10 \mathrm{~g}$ were obtained from the animal care center of Kerman Neuroscience Research Center. The animals were maintained at controlled condition, $25 \pm 1{ }^{\circ} \mathrm{C}$ and $12 \mathrm{~h}$ lightdark cycle and have access freely to standard chow diet and water. Our study was approved by the ethic committee of Kerman University of Medical Sciences, Kerman, Iran. P. atlantica resin oil which we have used in our study was prepared from Hakim-Tehrani Co. Kerman, Iran. Full compositions of $P$. atlantica oil have been reported previously. ${ }^{17,18}$

The animals were anesthetized by i.p. injection of Ketamin and Xylazine ( 60 and $4 \mathrm{mg}$ / $\mathrm{kg}$, respectively). The dorsal side of the animals were shaved and then induction of burn damage conducted by an aluminum plaque $(2.5 \times 2.5 \mathrm{~cm})$ on the shaved skin of rats for 15-20 seconds which was heated to $100^{\circ} \mathrm{C}$ to create a deep dermal burn wound. ${ }^{19,20}$ Duration of study was 14 days after burn injury and animals were divided into three groups $(\mathrm{n}=10)$ randomly as follow: Group 1 underwent just burn injury, Group 2 
underwent burn injury and received $200 \mathrm{mg} / \mathrm{kg} /$ day of $P$. atlantica resin oil topically, and Group 3 underwent burn injury and received $200 \mathrm{mg} /$ $\mathrm{kg} /$ day of sulfadiazine cream topically.

At the end of the study animals were anesthetized and sacrificed then burned skin were incised and separated. Samples were homogenized by Ultrasonic Processor (Hielscher, UP200H) in cold phosphate buffered saline (PBS, $\mathrm{pH}=7.4$ ) and then centrifuge at $4{ }^{\circ} \mathrm{C}$ and $15000 \mathrm{rpm}$ for 15 min. Supernatants were separated and aliquoted, then maintained at -80 until further experiments. Superoxide dismutase (SOD), Glutathione peroxidase (GPX), Total Antioxidant Status (TAS), Malondialdehyde (MDA), VEGF and hydroxyproline were measured in supernatant.

The wound contractions was reported as percent and were calculates by following formula: ${ }^{21} \%$ wound contraction= (wound area [day 1]-wound area [day 14]/wound area [day 1] $\times 100$

Measurement of VEGF, hydroxyprolin, MDA, TAS, SOD and GPX were conducted by specific kits (Hydroxyproline Elisa assay kit E0511Ra, Crystal Ray Biotech inc.; VEGF Elisa assay kit E0659Ra, Crystal Ray Biotech inc.; SOD, GPX and TAS conducted by using specific kits supplied from RANDOX laboratories Ltd. (TAS, Cat. No. NX2332; SOD, Cat. No. SD125; and GPX, Cat. No. RS505). MDA was measured as thiobarbituric acidreactive substances (TBARS) at $534 \mathrm{~nm}$ and 1,1,3,3-tetramethoxypropane was used to plot calibration curve. The data were expressed as mean \pm SEM. For comparison between groups One-way ANOVA test followed by post hoc Tukey's performed to compare mean differences between groups, and $p<0.05$ was considered as statistically significant.

\section{RESULTS}

Our results showed that $P$. atlantica resin oil significantly increase antioxidant defense, VEGF, hydroxyprolin and reduced MDA levels (Table 1, Figures 1 and 2). Also P. atlantica compared to sulfadiazine significantly increased

Table 1: Wound size, MDA and anti-oxidative parameters.

\begin{tabular}{llllll}
\hline Burn & $\begin{array}{l}\text { Wound contraction } \\
(\%)\end{array}$ & $\begin{array}{l}\text { MDA (nmol// } \\
\text { mg protein) }\end{array}$ & $\begin{array}{l}\text { SOD (U/mg } \\
\text { protein) }\end{array}$ & $\begin{array}{l}\text { GPX (U/mg } \\
\text { protein) }\end{array}$ & $\begin{array}{l}\text { TAS (mmol/ } \\
\text { mg protein) }\end{array}$ \\
\hline $\begin{array}{l}\text { Burn non-treated } \\
\text { control }\end{array}$ & $71.2 \pm 3.4$ & $6.1 \pm 0.4$ & $2.0 \pm 0.35$ & $7.9 \pm 0.07$ & $0.62 \pm 0.04$ \\
$\begin{array}{l}\text { Burn-treated with } P . \\
\text { atlantica }\end{array}$ & $98.6 \pm 2.5^{*}$ & $1.5 \pm 0.14^{*}$ & $6.0 \pm 0.43^{* \#}$ & $25.1 \pm 1.2^{* *}$ & $1.93 \pm 0.1^{* \#}$ \\
$\begin{array}{l}\text { Burn-treated with } \\
\text { sulfadiazine }\end{array}$ & $94.7 \pm 4.1^{*}$ & $2.2 \pm 0.22^{*}$ & $4.7 \pm 0.35^{*}$ & $19.0 \pm 1.18^{*}$ & $1.52 \pm 0.07^{*}$ \\
\hline
\end{tabular}

*Significant compared to control group, \#significant compared to sulfadiazine treated group, $(\mathrm{n}=10), p<0.05$ considered as statistically significant

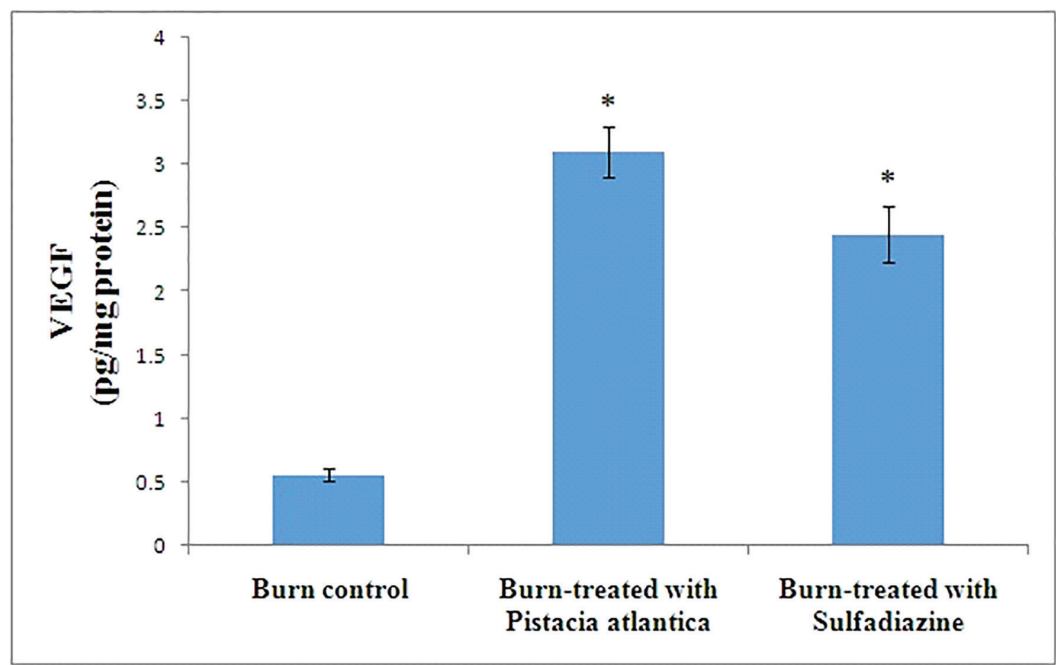

Fig. 1: VEGF levels in burn wound area. Group 1, burn control; group 2, burn-treated with $P$. atlantica resin oil topically; group 3, burn-treated with sulfadiazine. *statistically significant compared to Burn control group, \#statistically significant compared to burn-treated with sulfadiazine group. 


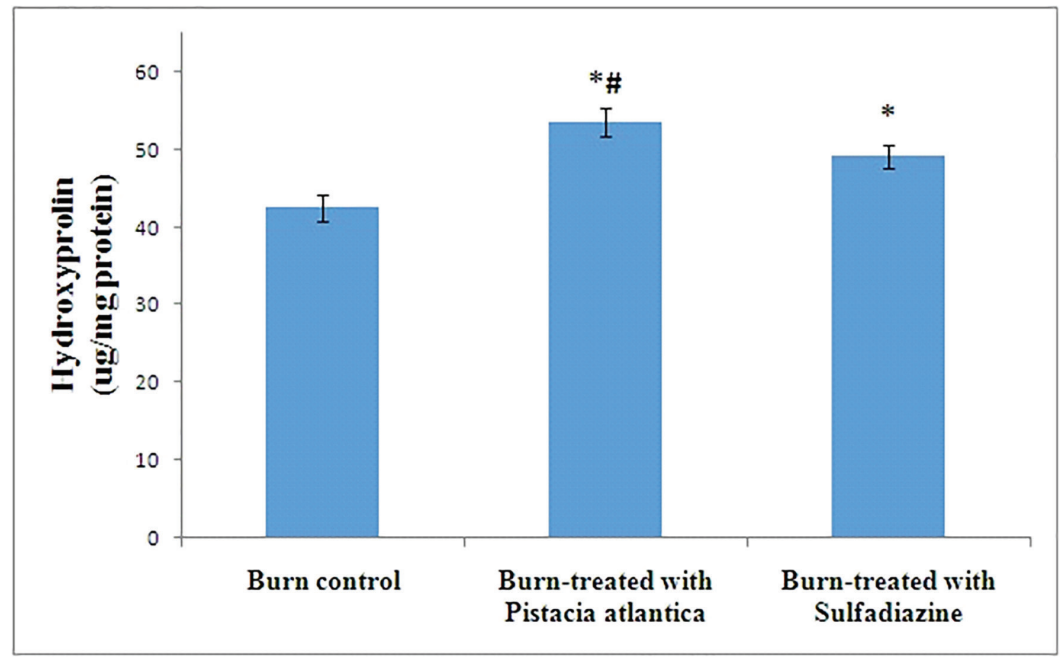

Fig. 2: Hydroxyproline levels in burn wound area. Group 1, burn control; group 2, burn-treated with P. atlantica resin oil topically; group 3, burn-treated with sulfadiazine. *statistically significant compared to Burn control group, \# statistically significant compared to burn-treated with sulfadiazine group.

SOD, GPX, TAS and hydroxyproline (Table 1, Figures 1 and 2). P. atlantica remarkably reduced wound size compared to burn control group (Table1)

\section{DISCUSSION}

Our results showed that Pistacia atlantica resin oil has remarkable antioxidant properties in experimentally induced- burn wound. Also we found that its resin oil is capable of increase the VEGF and hydroxyproline levels in wound area. Bozorgi et al. have reported that resin of $P$. atlantica has been used as treatment for some diseases and complicated condition such as digestive, hepatic, and kidney diseases. Also it demonstrated that the gum resin of $P$. atlantica can be used for wound healing and treatment of gastrointestinal problems. ${ }^{9}$

Previous studies have showed that $P$. atlantica resin oil have many compounds and $\alpha$-pinene (about $45-70 \%$ ) is the major components and it seems that $\alpha$-pinene is the effective substance present in resin oil., ${ }^{9,17,22,23}$ Also, Memariani et al. (2017) demonstrated that $\alpha$-pinene is the main component of $P$. atlantica oil and $2 \mathrm{~g} / \mathrm{kg}$ of $P$. atlantica oil was not harmful or toxic in vivo. They reported that $P$. atlantica oil showed protective effect against experimentally ethanol induced gastric ulcer. ${ }^{24}$ Also it has been reported that $\alpha$-Pinene showed significant low toxicity. ${ }^{18}$

$P$. atlantica oil antioxidative properties have been reported previously. ${ }^{12,16,25}$ Rezaie et al. have reported that $P$. atlantica oil has remarkable antioxidant activities compared with positive control. ${ }^{14}$ Koizumi et al. showed that severe burn damage cause promotion of free radicals which in turn result in vasodilatation and SOD can act as a protective factor against vasodilatation. ${ }^{26}$ Here we proved that $P$. atlantica resin oil significantly compared with burn control group elevated SOD levels.

We also found that $P$. atlantica resin oil is a potent antioxidant ointment which significantly improved antioxidant status of wound area in rats. Also we found that $P$. atlantica resin oil is rather potent than sulfadiazine to fight against free radicals present in burn wound. Farahpour et al. showed that $P$. atlantica hydroalcoholic hull extract showed antioxidant activity even higher than ascorbic acid, their finding are consistent with our antioxidative data about $P$. atlantica resin oil. ${ }^{22}$

It showed that $P$. atlantica resin oil has important antimicrobial activity against bacteria which showed resistance against some common antimicrobial drugs. ${ }^{15}$ Also other studies reported antimicrobial activity of $P$. atlantica oil. ${ }^{14,25}$ Therefore, $P$. atlantica resin oil in addition to increase antioxidants also has antimicrobial properties and it reduce the chance of infection and accelerate the process of wound healing.

Galiano et al. demonstrated that topical VEGF increased growth factor in damaged area and also recruits bone marrow derived cells which have remarkable role in wound repair. All together they showed that topical application of VEGF has wound healing properties. ${ }^{5}$ Haghdoost 
et al. showed that $P$. atlantica increases bFGF and PDGF and therefore result in angiogenesis. ${ }^{20}$ We showed that $P$. atlantica resin oil promotes VEGF levels in wound area in burn model injury in rats and this could explains its beneficial effects on wound contraction and repair in this study. Therefore, Galiano et al. and Haghdoost and colleagues reports are confirming our data regarding VEGF variations in wound area. ${ }^{5,20}$

Collagen is an extracellular matrix protein which is related to wound contraction and strength. Collagen turnover directly related to free hydroxyproline and quantification of hydroxyproline can be considered as a good parameter to monitor collagen turnover. ${ }^{22,27}$ Hamidi and colleagues showed that $P$. atlantica oil changed collagen pattern and caused organized collagen fiber after three weeks. ${ }^{28}$ Farahpour et $a l$. showed that $P$. atlantica hydroalcoholic hull extract as ointment increased hydroxyproline content and histological study showed that collagen score increased significantly. Also they found that $P$. atlantica hydroalcoholic hull extract promotes fibroblasts proliferation and therefore decline inflammation. ${ }^{22}$

IIango et al. evaluated methanol extract of L. acidissima on SOD, catalase, hydroxyproline, and epithelialization. They found that methanol extract of L. acidissima increase antioxidant SOD and also promotes hydroxyproline and epithelialization, therefore showed significant dose dependent wound healing activity. ${ }^{27} \mathrm{We}$ showed that $P$. atlantica resin oil increased hydroxyproline levels in wound area and this could be considered as a collagen turnover marker. Therefore, $P$. atlantica resin oil wound healing and contraction activity can be related to elevation of collagen turnover and consequently hydroxyproline.

Epithelialization is considered as an important factor which serves as a defining parameter of affluent wound contraction. ${ }^{28}$ Mehrabani et al. used $P$. atlantica oil in combination with three other oils from sesame (Sesamum indicum L.), hemp (Cannabis sativa L.) and walnut (Juglans regia L.) as a combined formula. This new formula increased wound contraction and accelerated epithelialization. ${ }^{21}$ Hamidi et al. showed that $P$. atlantica oil as gels ameliorated epithelialization which is as a marker of wound contraction. Also they showed that topical $P$. atlantica oil in rat with experimental wound cause biochemical and morphological promotion compared with control group. ${ }^{28}$

Therefore, according to these reports about $P$. atlantica oil we can anticipate that increased epithelialization and other beneficial histological changes can be considered as potential mechanism in addition to elevation of VEF and hydroxyproline and antioxidant status to increase. Finally according to previous studies $P$. atlantica resin oil has many beneficial properties and low toxicity, and our finding showed that Pistacia atlantica resin oil has antioxidant effects and increases VEGF and hydroxyproline, therefore, it seems that $P$. atlantica resin oil is a good, safe and potent candidate as a new therapeutic topical ointment for wound healing.

\section{ACKNOWLEDGEMENT}

The present article was financially supported by the Kerman University of Medical Sciences, Kerman, Iran.

\section{CONFLICT OF INTEREST}

The authors declare no conflict of interest.

\section{REFERENCES}

1 Mohtasham Amiri Z, Tanideh N, Seddighi A, Mokhtari M, Amini M, Shakouri Partovi A, Manafi A, Hashemi SS, Mehrabani D. The effect of Lithospermum officinale, silver sulfadiazine and alpha ointments in healing of burn wound injuries in rat. World J Plast Surg 2017;6:313-8.

2 Duci S, Arifi $\mathrm{H}$, Ahmeti H, Zatriqi V, Buja Z, Hoxha E, Mekaj A. Outcomes of older adults with burn injury: University Clinical Center of Kosovo. World J Plast Surg 2015;4:153-8.

3 Dai T, Huang YY, Sharma SK, Hashmi JT, Kurup DB, Hamblin MR. Topical Antimicrobials for Burn Wound Infections. Recent Pat Antiinfect Drug Discov 2010;5:124-51.

4 Drake DJ, Little CD. Exogenous vascular endothelial growth factor induces malformed and hyperfused vessels during embryonic neovascularization. Proc Natl Acad Sci 1995;92:7657-61.

5 Galiano RD, Tepper OM, Pelo CR, Bhatt KA, Callaghan M, Bastidas N, Bunting S, Steinmetz HG, Gurtner GC. Topical Vascular Endothelial Growth Factor Accelerates 
Diabetic Wound Healing through Increased Angiogenesis and by Mobilizing and Recruiting Bone Marrow-Derived Cells. Am J Pathol 2004;164:1935-47.

6 Dunphy JE, Udupa KN, Edwards LC. Wound Healing, A New Perspective with Particular Reference to Ascorbic Acid Deficiency. Ann Surg 1956;144:304-16.

7 Kalantar M, Goudarzi M, Foruozandeh H, Siahpoosh A, Khodayar MJ, Mahmoodi Koshkghazi S. The Topical Effect of Cappariss spinosa L. Extract on Burn Wound Healing. Jundishapur J Nat Pharm Prod 2016;5:1-7.

8 Afshar M, Ghaderi R, Zardast M, Delshad P. Effects of Topical Emu Oil on Burn Wounds in the Skin of Balb/c Mice. Dermatol Res Practice 2016;

9 Bozorgi M, Memariani Z, Mobli M, Salehi Surmaghi MH, Shams-Ardekani MR, Rahimi R. Five Pistacia species (P. vera, P. atlantica, P. terebinthus, P. khinjuk, and P. lentiscus): A review of their traditional uses, phytochemistry, and pharmacology. Sci World $J$ Volume 2013:219815:1-33.

10 M. Minaiyan M, Karimi F, Ghannadi A. Anti-inflammatory effect of Pistacia atlantica subsp. kurdica volatile oil and gum on acetic acid-induced acute colitis in rat. Res $J$ Pharmacogn 2015;2:1-12.

11 Delazar ARG, Reid RG, Sarker SD. GC/ MS Analysis of the Essential Oil from the Oleoresin of Pistacia atlantica Desf. subsp. atlantica from Algeria. Chem Natur Comp 2008;3:24-7.

12 Benhassainia H, Bendeddouchea FZ, Mehdadia Z, Romane A. Antioxidant activities and chemical composition of essential oil of Pistacia atlantica from Algeria. Natur Prod Comm 2010;31:929-33.

13 Taran M, Mohebali M, Esmaeli J. In Vivo Efficacy of Gum Obtained Pistacia Atlantica in Experimental Treatment of Cutaneous Leishmaniasis. Iran $J$ Public Health 2020;39:36-41.

14 Rezaie M, Farhoosh R, Sharifi A, Asili J, Iranshahi M. Chemical composition, antioxidant and antibacterial properties of Bene (Pistacia atlantica subsp. mutica) hull essential oil. J Food Sci Technol 2015;52:6784-90.

15 Ghalem BR, Mohamed B. Essential oil from gum of Pistacia atlantica Desf.: Screening of antimicrobial activity. Afr J Pharm Pharmacol
2009;3:87-91.

16 Peksel A, Arisan I, Yanardag R. Radical Scavenging and Anti-acetylcholinesterase Activities of Aqueous Extract of Wild Pistachio (Pistacia atlantica Desf.) Leaves. Food Sci Biotechnol 2013;22:515-22.

17 Barrero AF, Herrador MM, Arteaga JF, Akssira M, Mellouki F, Belgarrabe A. Chemical Composition of the Essential Oils of Pistacia atlantica Desf. J Ess Oil Res 2005;17:52-4.

18 Sharifi MS, Hazell SL. GC-MS Analysis and Antimicrobial activity of the essential oil of the trunk exudates from Pistacia atlantica kurdica. J Pharm Sci Res 2011;3:1364-7.

19 Abdel-Hamid AAM, Soliman MFM. Effect of topical aloe vera on the process of healing of full-thickness skin burn: a histological and immune histochemical study. J Histol Histopathol 2015;2:1-8.

20 Haghdoost F, Baradaran Mahdavi MM, Zandifar A, Sanei MH, Zolfaghari B, Haghjooy Javanmard S. Pistacia atlantica Resin Has a Dose-Dependent Effect on Angiogenesis and Skin Burn Wound Healing in Rat. Evid Based Complement Alternat Med 2013;893425:1-8.

21 Mehrabani M, Seyyedkazemi SM, Nematollahi MH, Jafari E, Mehrabani M, Mehdipour M. Accelerated Burn Wound Closure in Mice with a New Formula Based on Traditional Medicine. Iran Red Crescent Med J 2016;18:26613.

22 Farahpour MR, Mirzakhani N, Doostmohammadi J, Ebrahimzadeh M. Hydroethanolic Pistacia atlantica hulls extract improved wound healing process; evidence for mast cells infiltration, angiogenesis and RNA stability. Int J Surg 2015;17:88-98.

23 Bahmani M, Saki K, Asadbeygi M, Adineh A, Saberianpour S, Rafieian-Kopaei M. The effects of nutritional and medicinal mastic herb (Pistacia atlantica). J Chem Pharm Res 2015;7:646-53.

24 Memariani Z, Sharifzadeh M, Bozorgi M, Hajimahmoodi M, Farzaei MH, Gholami M, Siavoshi F, Saniee P. Protective effect of essential oil of Pistacia atlantica Desf. on peptic ulcer: role of $\alpha$-pinene. $J$ Tradit Chin Med 2017;37:57-63.

25 Benhammou N, Bekkara FA, Panovska TK. Antioxidant and antimicrobial activities of the Pistacia lentiscus and Pistacia atlantica 
extracts. Afr J Pharm Pharmacol 2008;2:22-8.

26 Koizumi T, Goto H, Tanaka H, Yamaguchi Y, Shimazaki S. Lecithinized superoxide dismutase suppresses free radical substrates during the early phase of burn care in rats. $J$ Burn Care Res 2009;30:321-8.

27 Ilango K, Chitra V. Wound Healing and Antioxidant Activities of the Fruit Pulp of Limonia
Acidissima Linn (Rutaceae) in Rats. Trop $J$ Pharmaceut Res 2010;9:223-30

28 Hamidi SA, Tabatabaeinejad A, Oryan A, Tabandeh MR, Tanideh N, Nazifi S. Cutaneous wound healing after topical application of pistacia atlantica gel formulation in rats. Turk J Pharm Sci 2017;14:65-74. 